PROCEEDINGS OF THE

AMERICAN MATHEMATICAL SOCIETY

Volume 43, Number 2, April 1974

\title{
LUSIN AND SUSLIN TOPOLOGIES ON A SET
}

\author{
KOHUR GOWRISANKARAN
}

\begin{abstract}
It is proved that the Borel subsets of two Suslin topologies on a set $X$ are identical if and only if there is a Suslin topology on $X$ finer than both the given topologies. Properties of the supremum topology are given under suitable conditions on the subdivisions of the spaces.
\end{abstract}

A Hausdorff topological space is said to be Lusin (resp. Suslin) if there is a continuous bijective mapping (resp. a continuous surjective mapping) from a complete separable metric space onto $X$. The topological spaces which are Suslin or Lusin have a number of interesting measure theoretic properties. We mention two of them here. The first and perhaps one of the very interesting properties is that if $X$ is a Lusin (resp. Suslin) space and $X$ is a subspace of a Hausdorff topological space $Y$, then $X$ is Borel in $Y$ (resp. $X$ is measurable for every finite Borel measure on $Y$ ). The second property is that every finite Borel measure on a Suslin space is a Radon measure. The development of the Suslin spaces and, in particular, the proofs of the above results can be found in [1].

In this note we consider a set $X$ with two Suslin topologies $\tau_{1}$ and $\tau_{2}$. It is of importance and interest to know when the $\tau_{1}$-Borel sets and $\tau_{2^{-}}$ Borel sets are identical. It is known that this happens if the $\inf \left(\tau_{1}, \tau_{2}\right)$ is a Hausdorff topology. We shall show that a necessary and sufficient condition for the above to happen is that $\sup \left(\tau_{1}, \dot{\tau}_{2}\right)$ is a Suslin topology (Theorem 1). We also prove some properties of the supremum topology in terms of conditions on subdivisions on the space.

We first have the

THEOREM 1. Let $\tau_{1}$ and $\tau_{2}$ be two Suslin (resp. Lusin) topologies on a set $X$. The $\tau_{1}$-Borel sets and $\tau_{2}$-Borel sets are identical if and only if the $\sup \left(\tau_{1}, \tau_{2}\right)$ is a Suslin (resp. Lusin) topology on $X$.

Proof. Assume that $\sup \left(\tau_{1}, \tau_{2}\right)$ is a Suslin topology on $X$. It is well known that the Borel sets of two comparable Suslin topologies on a set

Presented to the Society, September 1, 1972; received by the editors July 13, 1972.

AMS (MOS) subject classifications (1970). Primary 28A05; Secondary 54H05, 04A15.

Key words and phrases. Lusin, Suslin, topology, Borel sets, Radon space, Radon measures.

(c) American Mathematical Society 1974 
are identical [1]. We deduce that $\sup \left(\tau_{1}, \tau_{2}\right), \tau_{1}$ and $\tau_{2}$ all have the same Borel sets. Conversely, assume $\tau_{1}$-Borel sets and $\tau_{2}$-Borel sets are identical. Let $\Delta$ be a diagonal in $X \times X$. Then, $\Delta$ is closed in the product space $\left(X \times X, \tau_{1} \times \tau_{1}\right)$. Also the complement of $\Delta$ which is $\tau_{1} \times \tau_{1}$ open is Lindelöf and hence can be covered by a countable number of $\tau_{1} \times \tau_{1}$ open rectangles of the form $V \times W$. However, $W$ is $\tau_{2}$-Borel and each such set $V \times W$ is $\tau_{1} \times \tau_{2}$-Borel. It follows that $(X \times X) \mid \Delta$ is $\tau_{1} \times \tau_{2}$-Borel and hence $\Delta$ is $\tau_{1} \times \tau_{2}$-Borel. But since $\left(X \times X, \tau_{1} \times \tau_{2}\right)$ is Suslin (resp. Lusin), we deduce that $\Delta$ with the induced $\tau_{1} \times \tau_{2}$-topology is Suslin (resp. Lusin). Also, $X$ with the $\sup \left(\tau_{1}, \tau_{2}\right)$-topology is homeomorphic to $\Delta$ with the induced $\tau_{1} \times \tau_{2}$-topology. The proof is complete.

Corollary For $X, \tau_{1}, \tau_{2}$ as above, if $\inf \left(\tau_{1}, \tau_{2}\right)$ is Hausdorff the $n$ $\sup \left(\tau_{1}, \tau_{2}\right)$ is a Suslin topology on $X$.

The Corollary is deduced as an immediate consequence by observing that under the additional hypothesis $\tau_{1}$-Borel sets and $\tau_{2}$-Borel sets are identical.

We now recall

Definition 1. Let $(X, \tau)$ be a Hausdorff topological space. A subdivision $\left\{\left(\mathscr{C}_{n}, p_{n}, \varphi_{n}\right)\right\}$ on $X$ is the following system.

(a) $\mathscr{C}_{n}$, for every $n=0$ to $\infty$, is a countable set and $p_{n}$ is a surjection from $\mathscr{C}_{n+1}$ to $\mathscr{C}_{n}$.

(b) For every $n=0$ to $\infty, \varphi_{n}$ is a one-one mapping of $\mathscr{C}_{n}$ into the collection of nonvoid subsets of $X$ such that $\bigcup\left\{\varphi_{0}(c): c \in \mathscr{C}_{0}\right\}=X$ and for every $n \in N$ and every $c \in \mathscr{C}_{n}, \bigcup\left\{\varphi_{n+1}(d): d \in \mathscr{C}_{n+1}, p_{n}(d)=c\right\}=\varphi_{n}(c)$.

(c) For every coherent sequence $\left\{c_{n}\right\}$ viz. $c_{n} \in \mathscr{C}_{n}$ such that $p_{n}\left(c_{n}\right)=c_{n-1}$, by (b), $\left\{\varphi_{n}\left(c_{n}\right)\right\}$ forms a base of a filter and this filter converges to an element $x$ in $X$ such that $x$ belongs to $\varphi_{n}\left(c_{n}\right)$ for each $n$.

(d) Every element $x$ in $X$ is the limit of at least one filter as in (c).

Further the subdivision $\left\{\left(\mathscr{C}_{n}, p_{n}, \varphi_{n}\right)\right\}$ is said to be a strict subdivision if for every $n,\left\{\varphi_{n}(c)\right\}_{c \in \mathscr{C}_{n}}$ is a sequence of mutually disjoint subsets of $X$.

The following theorem is a well-known result about Suslin and Lusin spaces [1].

TheOrem S. A Hausdorff topological space $(X, \tau)$ is Suslin (resp. Lusin) if and only if $(X, \tau)$ admits a subdivision (resp. a strict subdivision).

Definition 2. Let $(X, \tau)$ be a topological space and $\left\{\left(\mathscr{C}_{n}, p_{n}, \varphi_{n}\right)\right\}$ a subdivision on $X$. The topology on $X$ with the subbase for open sets consisting of $\left\{\varphi_{n}(c): c \in \mathscr{C}_{n}, n \in N\right\}$ is called the topology associated with the subdivision. 
Lemma 1. Let $(X, \tau)$ be a Lusin space with a strict subdivision $\left\{\left(\mathscr{C}_{n}, p_{n}, \varphi_{n}\right)\right\}$. Then the topology $\tau^{\prime}$ associated with this subdivision is the finest among the topologies on $X$ for which $\left\{\left(\mathscr{C}_{n}, p_{n}, \varphi_{n}\right)\right\}$ is a subdivision. Consequently $\left(X, \tau^{\prime}\right)$ is Lusin and $\tau$-Borel sets and $\tau^{\prime}$-Borel sets are identical.

Proof. Let $\alpha$ be any Hausdorff topology on $X$ such that $\left\{\left(\mathscr{C}_{n}, p_{n}, \varphi_{n}\right)\right\}$ is a subdivision for $(X, \alpha)$. Then, by the condition (d) of the definition of subdivision, given $x$ in $X$ and an $\alpha$-neighborhood $V$ of $x$, there is a $c \in \mathscr{C}_{n}$ such that $x$ is in $\varphi_{n}(c)$ and $\varphi_{n}(c) \subset V$. Hence, the topology $\tau^{\prime}$ is finer than $\alpha$ on $X$. Since there is at least one such topology viz. $\tau$, we conclude that $\left(X, \tau^{\prime}\right)$ is Hausdorff.

Now since the subdivision $\left\{\left(\mathscr{C}_{n}, p_{n}, \varphi_{n}\right)\right\}$ is strict, we observe that $\left\{\varphi_{n}(c): c \in \mathscr{C}_{n}, n \in N\right\}$ is in fact, a base for the topology $\tau^{\prime}$. Also, for any $x$ in $X$ there is exactly one coherent sequence $\left\{c_{n}\right\}$ such that $\varphi_{n}\left(c_{n}\right)$ contains $x$ for every $n$. Clearly, $\left\{\varphi_{n}\left(c_{n}\right)\right\}$ for this coherent sequence is precisely a base of $\tau^{\prime}$-neighborhoods of $x$ and we conclude that condition (d) of the definition of subdivision is satisfied by $\left\{\left(\mathscr{C}_{n}, p_{n}, \varphi_{n}\right)\right\}$ relative to $\left(X, \tau^{\prime}\right)$. On the other hand, given any coherent sequence $\left\{c_{r}\right\}$ there is a unique $x$ in $X$ which is contained in all $\varphi_{n}\left(c_{n}\right)$ and to which $\left\{\varphi_{n}\left(c_{n}\right)\right\}$ converges in the $\tau$-topology. By the definition, any $\tau^{\prime}$-neighborhood of $x$ necessarily contains $\varphi_{m}\left(c_{m}\right)$ for some $m$ and we conclude that $\left\{\varphi_{n}\left(c_{n}\right)\right\}$ converges to $x$ in $\tau^{\prime}$. Hence $\left\{\left(\mathscr{C}_{n}, p_{n}, \varphi_{n}\right)\right\}$ is a strict subdivision for $\left(X, \tau^{\prime}\right)$. The proof of the Lemma is now completed easily.

THEOREM 2. Let $\tau_{1}$ and $\tau_{2}$ be two topologies on a set $X$ such that $\left(X, \tau_{1}\right)$ and $\left(X, \tau_{2}\right)$ are Lusin spaces. Then the following are equivalent:

(2.1) There is a topology $\tau$ on $X$ finer than both $\tau_{1}$ and $\tau_{2}$ such that $(X, \tau)$ is a Lusin space.

(2.2) There are strict subdivisions $\left\{\left(\mathscr{C}_{n}, p_{n}, \varphi_{n}\right)\right\}$ and $\left.\left\{\mathscr{D}_{n}, q_{n}, \psi_{n}\right)\right\}$ respectively for the spaces $\left(X, \tau_{1}\right)$ and $\left(X, \tau_{2}\right)$ satisfying: For every pair of points $x, y$ in $X, x \neq y$, there are disjoint sets $\varphi_{m}\left(c_{m}\right)$ and $\psi_{n}\left(d_{n}\right)$ such that $x$ is in $\varphi_{m}\left(c_{m}\right)$ and $y$ is in $\psi_{n}\left(d_{n}\right)$.

(2.3) There is a common strict subdivision for the two spaces $\left(X, \tau_{1}\right)$ and $\left(X, \tau_{2}\right)$.

Proof. Clearly $(2.3) \Rightarrow(2.2)$. Now, suppose there are subdivisions satisfying the condition (2.2). Let $\tau_{1}^{\prime}$ and $\tau_{2}^{\prime}$ be the topologies associated with these subdivisions. Clearly $\left(X \times X, \tau_{1}^{\prime} \times \tau_{2}^{\prime}\right)$ is a Lusin space and for every $(x, y), x \neq y$, there is a $\tau_{1}^{\prime} \times \tau_{2}^{\prime}$ open set of the form $\varphi_{m}\left(c_{m}\right) \times \psi_{n}\left(d_{n}\right)$ which contains $(x, y)$ and does not intersect the diagonal $\Delta$. It follows that $\Delta$ is $\tau_{1}^{\prime} \times \tau_{2}^{\prime}$ closed and is hence a Lusin space with the induced topology. 
However, $\Delta$ with this topology is homeomorphic to $X$ with the $\sup \left(\tau_{1}, \tau_{2}\right)$ topology. This shows that $(2.2) \Rightarrow(2.1)$. Finally, if $(2.1)$ is satisfied then any strict subdivision of a topology finer than both $\tau_{1}$ and $\tau_{2}$ is also a common strict subdivision for both $\tau_{1}$ and $\tau_{2}$. The proof is complete.

We have the following result for Suslin spaces with analogous conditions.

THEOREM 3. Let $\tau_{1}$ and $\tau_{2}$ be two topologies on a set $X$ such that $\left(X, \tau_{1}\right)$ and $\left(X, \tau_{2}\right)$ are Suslin spaces. Let there be subdivisions $\left\{\left(\mathscr{C}_{n}, p_{n}\right.\right.$, $\left.\left.\varphi_{n}\right)\right\}$ for $\left(X, \tau_{1}\right)$ and $\left\{\left(\mathscr{D}_{m}, q_{m}, \psi_{m}\right)\right\}$ for $\left(X, \tau_{2}\right)$ such that given $x, y$ in $X$, $x \neq y$, there are disjoint sets $\varphi_{m}\left(c_{m}\right)$ containing $x$ and $\psi_{n}\left(d_{n}\right)$ containing $y$. Then $X$ with the $\sup \left(\tau_{1}, \tau_{2}\right)$-topology is a Radon space; that is, every finite Borel measure on this space is Radon.

Proof. Let $\tau_{1}^{\prime}$ and $\tau_{2}^{\prime}$ be the topologies associated with the subdivisions. We observe that $\tau_{1}^{\prime}$ (resp. $\tau_{2}^{\prime}$ ) is finer than $\tau_{1}$ (resp. $\tau_{2}$ ) and both the topologies $\tau_{1}^{\prime}$ and $\tau_{2}^{\prime}$ have countable bases for open sets. Also, as in the proof of the earlier theorem, the given condition on the subdivision implies that the diagonal $\Delta$ is $\tau_{1}^{\prime} \times \tau_{2}^{\prime}$ closed in $X \times X$. Let $\mathscr{B}(Y, \tau)$ denote the $\sigma$-algebra of Borel subsets of any topological space $(Y, \tau)$ and $\mathscr{M}(Y, \tau)$ the $\sigma$-algebra of all subsets of $Y$ that are measurable for every finite measure on $\mathscr{B}(Y, \tau)$. Now, every Radon space is universally measurable as a subspace of any topological space and this is in particular true for Suslin spaces. We conclude that

and

$$
\mathscr{B}\left(X, \tau_{1}\right) \subset \mathscr{B}\left(X, \tau_{1}^{\prime}\right) \subset \mathscr{M}\left(X, \tau_{1}\right),
$$

$$
\mathscr{B}\left(X, \tau_{2}\right) \subset \mathscr{B}\left(X, \tau_{2}^{\prime}\right) \subset \mathscr{M}\left(X, \tau_{2}\right) .
$$

Let $A_{k}$ be in $\mathscr{M}\left(X, \tau_{k}\right)$ for $k=1,2$. Then $A_{k}$ with the induced $\tau_{k}$-topology is Radon and further every $\tau_{k}$-compact subset $E_{k}$ of $A_{k}$ is metrizable since $E_{k}$ is a compact subset of the Suslin space $\left(X, \tau_{k}\right)$. This shows that $A_{1} \times A_{2}$ with $\tau_{1} \times \tau_{2}$-topology is Radon [1]. We deduce easily that

$$
\mathscr{M}\left(X, \tau_{1}\right) \otimes \mathscr{M}\left(X, \tau_{2}\right)
$$

is contained in $\mathscr{M}\left[X \times X, \tau_{1} \times \tau_{2}\right]$. Further, since $\left(X, \tau_{1}^{\prime}\right)$ and $\left(X, \tau_{2}^{\prime}\right)$ both have countable basis for open sets we conclude that

$$
\mathscr{B}\left(X, \tau_{1}^{\prime}\right) \otimes \mathscr{B}\left(X, \tau_{2}^{\prime}\right)=\mathscr{B}\left(X \times X, \tau_{1}^{\prime} \times \tau_{2}^{\prime}\right) .
$$

It follows that

$$
\Delta \in \mathscr{B}\left(X \times X, \tau_{1}^{\prime} \times \tau_{2}^{\prime}\right) \subset \mathscr{M}\left(X \times X, \tau_{1} \times \tau_{2}\right) .
$$

Hence, $\Delta$ is measurable for every finite Borel measure on $\left(X \times X, \tau_{1} \times \tau_{2}\right)$. However, this latter space is Radon and it follows that $\Delta$ with the induced 
$\tau_{1} \times \tau_{2}$-topology is a Radon space [1]. Since $X$ with $\sup \left(\tau_{1}, \tau_{2}\right)$-topology is homeomorphic to $\Delta$ with the induced $\tau_{1} \times \tau_{2}$-topology, we conclude that $X$ with the $\sup \left(\tau_{1}, \tau_{2}\right)$-topology is a Radon space, completing the proof.

\section{BIBLIOGRAPHY}

1. L. Schwartz, Radon measures on arbitrary topological spaces and cylindrical measures, Tata Institute of Fundamental Research Monograph, Oxford Univ. Press, Bombay, 1973.

Mathematics Department, McGill University, Montréal 101, QuébeC 